\title{
Ciclos económicos e inmigración: trabajadoras latinoamericanas en Estados Unidos durante la Gran Recesión *
}

\author{
Business cycles and immigration: Latino female workers in United States \\ through the Great Recession
}

\author{
Marina Ariza \\ Instituto de Investigaciones Sociales, Universidad Nacional Autónoma de México
}

Recibido: 22 de mayo de 2017. Aprobado: 7 de septiembre de 2017.

\begin{abstract}
Resumen
A pesar de haber afectado más a los hombres trabajadores que a las mujeres, la primera crisis global del siglo XXI es un escenario adecuado para analizar la compleja relación entre ciclos económicos, fuerza de trabajo femenina e inmigración. Con base en metodologías cuantitativas se emprende una evaluación del impacto de la Gran Recesión sobre la fuerza de trabajo inmigrante latinoamericana en Estados Unidos, contrastando su situación respecto de las nativas y el resto de las inmigrantes en tres momentos analíticos: 2007 (precrisis), 2009 (recesión) y 2014 (recuperación). Los resultados resaltan tanto el carácter mayormente contracíclico (de amortiguación) de las trabajadoras inmigrantes (en particular latinoamericanas) a lo largo del ciclo económico — mediado

Este artículo es producto de la investigación "Dinámica económica y migración femenina en un entorno recesivo: mujeres latinoamericanas en el mercado de trabajo estadounidense, 2006-2014” y cuenta con financiamiento del Programa de Apoyo a Proyectos de Investigación e Innovación Tecnológica (PAPIIT), de la Universidad Nacional Autónoma de México (proyecto IN302816). La autora agradece al IPUMS (Integrated Public Use Microdata Series International), de la Universidad de Minnesota, EUA, el acceso a las encuestas en que se basó este estudio, como también a la Unidad de Investigación Social y Aplicada de Estudios de Opinión del Instituto de Investigaciones Sociales de la UNAM, en la persona de la Dra. Sandra Murillo. Una versión preliminar de este trabajo fue presentada en el VII Congreso de la Asociación Latinoamericana de Población, en 2016.

* Dra. en Ciencia Social con especialidad en Sociología por El Colegio de México, investigadora titular del Instituto de Investigaciones Sociales de la Universidad Nacional Autónoma de México, Circuito Mario de la Cueva s/n, Universidad Nacional Autónoma de México, Ciudad de México, C.P. 04510.ariza@unam.mx
\end{abstract}


por la estructura segregada de los mercados de trabajo-, como los perdurables efectos negativos del fuerte período recesivo sobre sus posibilidades de empleo a varios años de iniciada la recuperación económica.

Palabras clave: ciclos económicos, fuerza de trabajo femenina, inmigración.

\begin{abstract}
Despite having affected the male population more than the female population, the first global crisis of the 21st century provides a useful scenario for analyzing the complex relationship between economic cycles, the female labor force and immigration. Quantitative methodologies were used to evaluate the impact of the Great Recession on the Latina immigrant labor force in the United States, contrasting its situation with that of US-born female workers and other female immigrants at three points in time: 2007 (precrisis), 2009 (recession), and 2014 (recovery). The results highlight both the predominantly countercyclical (buffering) nature of female immigrant workers (particularly Latinas) throughout the economic cycle - mediated by the gender segregation of labor markets and the lasting negative effects of the deep recession on their employment opportunities several years after the start of the economic recovery.
\end{abstract}

Keywords: business cycles, female labor force, immigration.

\title{
Introducción
}

El objetivo de este artículo es describir los efectos de la Gran Recesión y el subsecuente período de escaso dinamismo económico que le sucedió, sobre la fuerza de trabajo inmigrante latinoamericana. Para ello se toman tres momentos de observación: el año 2007, punto de referencia previo a la crisis; el año $2009^{1}$, momento que condensa la peor

De acuerdo con el National Bureau of Economic Research (NBER), oficialmente la recesión inició en diciembre de 2007 y habría concluido en junio de 2009 (http://www.nber.org/cycles/US_Business_Cycle_Expansions_ and_Contractions_20120423.pdf). La fuente de información en que nos basamos, la Current Population Survey (Encuesta Continua de Población, suplemento de marzo), permite una buena observación para cada momento 
parte del interludio recesivo; y 2014, punto de observación que por su distancia con la fase más aguda debería recoger los efectos de la recuperación.

En los años transcurridos desde que se abriera paso la primera crisis económica global del siglo XXI, mucho se ha escrito sobre sus perniciosas consecuencias en los niveles de vida de la población, su irrupción como expresión de los límites estructurales del capitalismo y el paralelismo que guarda con intervalos recesivos precedentes, el más aludido de los cuales es la Gran Depresión de 1929² (Eichengreen, 2015; Knoop, 2015; Solimano, 2015). La referencia a ese otro gran cisma económico global no es fortuita: su magnitud y devastadoras consecuencias suscitan más de una remembranza. Entre otras cosas, ha sido la de mayor duración (dieciocho meses) desde la Gran Depresión y ha sobrepasado en pérdida de empleos a las cinco recesiones previas (Fligstein y Goldstein, 2011; Grusky, Wimer y Western, 2011).

Uno de los rasgos destacados entre los analistas de la reciente crisis económica ha sido el mayor impacto negativo sobre la fuerza de trabajo masculina, al grado de que ha sido denominada por algunos como the man-cession (Folbre, 2010; Hout, Levanon y Cumberworth, 2011; Rampell, 2009); si bien hay quienes consideran desafortunada esta adjetivación (Hartman, 2009). El efecto diferencial de los altibajos económicos sobre la fuerza de trabajo masculina y femenina está mediado por la segregación por sexo como rasgo estructural de los mercados de trabajo, y puede variar a lo largo de la evolución del ciclo (Rubery y Rafferty, 2013). La alta concentración de los hombres en sectores muy sensibles a los vaivenes económicos — como la construcción por ejemploeleva su vulnerabilidad ante eventuales shocks económicos, sean estos endógenos o exógenos (Martin, 2009). El acentuado énfasis en el impacto sobre la fuerza de trabajo masculina ha dejado a la sombra la comprensión cabal de las consecuencias sobre las mujeres trabajadoras; en particular las inmigrantes, una parte de la cuales ha exhibido tradicionalmente condiciones laborales bastante más precarias que los hombres (Caicedo, 2010). Mediante el contraste sistemático de indicadores laborables clave en los tres momentos de observación (2007, 2009 y 2014), este trabajo pretende arrojar luz sobre los efectos diferenciales del largo período recesivo inaugurado en diciembre de 2007 sobre la fuerza de trabajo femenina inmigrante, tomando en cuenta su heterogeneidad.

elegido: en marzo de 2007 la recesión no había iniciado, mientras que en marzo de 2009 llevaba alrededor de quince meses.

2 En términos históricos, el crack de 1929 marca el relevo en la hegemonía mundial desde Gran Bretaña a Estados Unidos, entonces como ahora epicentro del colapso financiero cuyas ondas expansivas tuvieron también un alcance global (Solimano, 2015). 
El texto consta de tres apartados. En el primero se reseñan los aspectos más destacables del prolongado intervalo recesivo en su conexión con los mercados de trabajo y la inmigración. En el segundo se describen - con base en un análisis bivariado- los principales efectos sobre la población femenina inmigrante trabajadora. En el tercero y último se analizan los resultados del ajuste de un conjunto de modelos estadísticos para medir la chance de caer en el desempleo en cada uno de los tres momentos de observación contemplados (2007, 2009 y 2010), a partir de un conjunto de variables independientes.

\section{La Gran Recesión: ciclos económicos e inmigración}

Como es sabido, las variaciones en la actividad económica a través del tiempo - los ciclos económicos - son inherentes al funcionamiento de la economía capitalista (Heath, $2012)^{3}$. De 1900 a la fecha, Estados Unidos ha experimentado en total veintidós episodios recesivos (con sus subsiguientes episodios de recuperación), incluyendo el que nos ocupa (Hout y Cumberth, 2012) ${ }^{4}$. Análogamente a otros momentos de gran calado, la Gran Recesión fue catapultada por una crisis financiera a la que precedió un auge extraordinario en el sector de la construcción de viviendas y edificios, y un endeudamiento excesivo en la modalidad de hipotecas de alto riesgo (Solimano, 2015) .

En el mismo sentido en que existen paralelismos históricos destacables en los factores que desencadenan las fases recesivas, los hay también en cuanto a las respuestas del sector productivo y las consecuencias para la población trabajadora. Ante el aumento extraordinario de la capacidad ociosa y la sobreproducción de bienes como síntomas que

3 En estricto sentido, el ciclo económico o "business cycle" refiere a las fluctuaciones en la producción o en la actividad económica que se prolongan desde un par de trimestres a varios años. Consiste en expansiones simultáneas en la mayoría de las actividades económicas, seguidas de recesiones generalizadas de forma similar, de magnitud y carácter similares, que no son divisibles en ciclos más cortos (Burns y Mitchell, 1946, citado por Heath, 2012, p. 32).

4 De acuerdo con el NBER, por recesión se entiende: "Una caída significativa de la actividad económica que se extiende por toda la economía en su conjunto, que dura más que unos pocos meses y que es normalmente visible en el PIB real, el ingreso real, el empleo, la producción industrial y en las ventas al menudeo y mayoreo". Dicha institución considera un umbral de al menos seis meses para declarar la existencia de una recesión, aunque se ha popularizado como parámetro el que abarque dos trimestres consecutivos.

5 El autor destaca que este sector fue también el detonante de la crisis en Islandia, en 2008, y en España, entre 20092010. Refiere que durante el siglo XIX burbujas semejantes precedieron crisis de gran alcance en las economías centrales, la más importante de las cuales fue quizás la Larga Depresión, en 1873, con epicentro en Viena y Alemania. Dicha depresión estuvo precedida por un auge en el sector de la construcción de rieles (Solimano, 2015, p. 107). 
anteceden al cisma económico, se emprenden recortes sustantivos en la producción y el empleo. Son típicamente los sectores productivos (minería, industria de la construcción y manufactura) los primeros en acometer los ajustes, pues la evaluación periódica de los inventarios los alerta tempranamente acerca de la acumulación desfavorable de stocks. En el momento más agudo de la crisis - en el lapso comprendido entre el último cuatrimestre de 2007 y el segundo de 2009-, el PIB se contrajo por encima del 5\%, a la par que el desempleo escaló a dos dígitos (10\%) (Martin, 2009; Rothstein, 2012). En términos absolutos, esto representó una pérdida de 5,9 millones de puestos de trabajo entre el otoño de 2007 y la primavera de 2009, y la incorporación de 5,7 millones de personas al desempleo.

El incremento inusual en los niveles de desocupación es a todas luces uno de los correlatos más evidentes de la crisis, dado el papel central del mercado de trabajo en la dinámica económica (Knoop, 2015). Los valores alcanzados en el contexto de la Gran Recesión hicieron converger por primera vez a la economía estadounidense con varias de las europeas, cuyas cifras a lo largo de los últimos veinte años han estado consistentemente por encima de la media de los países de la OCDE (Papademetriou et al., 2010) ${ }^{6}$. La permanencia de tasas de desempleo excepcionalmente altas en 2014 -superiores al menos a las de 2007 - a varios años de haber iniciado la fase de recuperación (crecimiento del producto), aunada a la importancia que ha cobrado el desempleo de larga duración, se revelan como uno de los rasgos singulares del ciclo económico inaugurado en diciembre de 2007. La Gran Recesión reedita por segunda vez (aunque a mayor profundidad) la paradoja de crecimiento del producto (que remontó su curso en junio de 2010), sin creación de empleo (jobless recovery) ${ }^{7}$, lo que denota la profundidad del período recesivo y - desde la perspectiva de algunos- sugiere efectos transformativos de mayor calado cuyo sentido aún no es posible avizorar (Hout, et al., 2011) ${ }^{8}$.

En contraste con lo que acontece en el sector productivo, el sector servicios es bastante menos sensible a los vaivenes del ciclo. En una coyuntura recesiva puede incluso ampliarse, ya sea de forma "espontánea" o como producto de políticas expresamente contra cíclicas encaminadas a paliar las secuelas del quiebre económico (Milkman, 1976;

6 En la historia reciente de Estados Unidos, solo hubo otra ocasión en que la tasa de desempleo se colocó por encima de 10\% (1979-1983), y lo hizo a lo largo de treinta y ocho meses, es decir, en más de tres años. Durante la Gran Recesión, la tasa se duplicó en tan solo veintiséis meses (Hout, et al., 2011).

7 Según refiere Hout (2012), desde 1948 la recuperación del empleo no habría sido tan lenta. La recesión de 20012003 habría sido la primera de estas características desde la posguerra.

8 Para Rothstein (2012), dicha debilidad obedece principalmente a la insuficiencia de la demanda en el período postcrisis. 
Rubery y Rafferty, 2013). En efecto, los servicios de salud - uno los subsectores más voluminosos en términos de empleo-crecieron por encima del 1\% en los primeros años del colapso financiero (Rothstein, 2012) ${ }^{9}$. Son estos factores los que tornan inteligible el mayor efecto relativo sobre la población ocupada masculina en los momentos iniciales de la crisis, comportamiento que en sentido general ha caracterizado a la mayoría de las recesiones ocurridas en Estados Unidos durante los siglos XX y XXI, refrendado de igual modo en la Gran Recesión ${ }^{10}$. Así, por ejemplo, mientras en la fase más aguda del intervalo recesivo la participación económica de los hombres en el mercado de trabajo estadounidense se contrajo, la femenina permaneció relativamente estable o bien mostró una modesta reversión. Un comportamiento semejante ha sido descrito en España, en el que las mujeres - sobre todo las latinoamericanas - sufrieron menores pérdidas relativas de empleo que los hombres (Parella, 2015). Este aspecto invita a examinar con precaución la relación entre fuerza de trabajo femenina y dinámica del ciclo económico (valles y crestas) (Humphries, 1988), y sugiere que al menos en los momentos iniciales de la Gran Recesión habrían jugado un rol contracíclico (Hartman, 2009; Hout, 2012) ${ }^{11}$. En el análisis que emprenden del impacto de la recesión en la población femenina trabajadora en Reino Unido, Rubery y Rafferty (2013) concluyen, que: 1) a contrapelo de lo ocurrido en otras coyunturas económicas semejantes, en esta ocasión - y a contracorriente de los hombres - las mujeres no transitaron hacia la inactividad económica; 2) la expansión del empleo público en la fase inicial de la crisis les otorgó cierta protección, misma que quedó sin efecto en el subsecuente período de austeridad económica.

Estudios anteriores realizados para el Reino Unido y Estados Unidos mostraron que la volatilidad cíclica de las mujeres concernía principalmente a un subconjunto de industrias manufactureras y no era observable ni en el total del empleo ni en aquellos sectores en donde su presencia era proporcionalmente baja con relación a la masculina (Humphries, 1988; Rubery y Tarling, 1988). En un nivel agregado, el empleo femenino tendía a crecer en virtud de que las mujeres se encontraban sobrerrepresentadas en subsectores relativamente protegidos del mercado de trabajo (servicios calificados) vis a vis, los más afectados como podría ser la industria manufactura, por ejemplo. Este juego de vasos comunicantes ocultaba en cierto modo el desempeño de las mujeres en

9 Otros sectores que se expandieron fueron la minería, que absorbe un porcentaje relativamente pequeño de trabajadores, y el empleo público, si bien modestamente (Rothstein, 2012).

10 De acuerdo con Hartman (2009), la de 2001 fue la única recesión en la cual el empleo femenino cayó simultáneamente con el masculino, con una pérdida a la sazón de 230.000 empleos a lo largo de la misma. En las cinco recesiones ocurridas entre 1969 y 1991, los hombres han perdido más empleo que las mujeres (Levine, 2009).

11 Durante la Gran Depresión, otro momento paradigmático en la historia de los ciclos económicos, el empleo femenino creció, a la par que disminuyó el masculino (Levine, 2009). 
subsectores económicos específicos. Tales aspectos sugieren que una comprensión cabal de los efectos de los ciclos económicos sobre el empleo femenino y su comportamiento a lo largo de sus cénit y simas, amerita de un análisis cuidadoso a diferentes escalas de observación, pues el balance intra e intersectorial dista de ser una suerte de suma cero.

Además de los hombres, entre los grupos poblacionales más vulnerables a los embates económicos en el contexto estadounidense figuran los afroamericanos y los hispanos, en los que las tasas de desempleo y los niveles de pobreza escalaron de forma vertiginosa (Hout, Levanon y Cumberworth, 2011; Papademetriou y Terrazas, 2010), en parte por tratarse - como es sabido- de grupos con condiciones preexistentes de vulnerabilidad. En referencia a la experiencia de los latinoamericanos, algunos autores se preguntan si puede hablarse de una Hispanic Recession, evocando una suerte de paralelo con la Mancession (Papademetriou y Terrazas, 2010, p. 57). Entre otros aspectos, se destaca que el desempleo, el subempleo y los trabajadores desalentados crecieron de forma dramática en ellos, en contraste con los nativos y los asiáticos. En la mirada de varios autores, de haber tenido lugar, la Hispanic Recession sería atribuible a una combinación de factores sociodemográficos e institucionales. Los primeros aludirían a la menor dotación de capital humano de los latinos (en especial los mexicanos), su menor edad promedio - visto que la crisis se cebó sobre los segmentos más jóvenes del mercado trabajo-, así como su mayor masculinización relativa, pues es sabido que el colectivo de los inmigrantes hispanos está hegemonizado por los de origen mexicano, un flujo (y un stock) en el que predominan los hombres (Papademetriou y Terrazas, 2010). Entre los factores institucionales, los autores destacarían lo que Cachón y Aysa-Lastra (2015) llaman un marco institucional discriminatorio, que en el caso particular de buena parte de los inmigrantes hispanos de origen mexicano los confina al estatus legal de indocumentados, esto es, de trabajadores carentes de derechos y prerrogativas básicos; trabajadores a la sombra.

En sentido general, la mayor vulnerabilidad de los inmigrantes emana de un conjunto de aspectos interrelacionados que los coloca en una situación desfavorable en el mercado de trabajo: su sobrerrepresentación entre los sectores menos calificados de la fuerza de trabajo, su concentración en los ámbitos productivos más reactivos a la desaceleración económica; su escaso capital humano en términos de experiencia y/o conocimiento del idioma; las dificultades para lograr la efectiva transferibilidad de sus conocimientos y experiencia (procesos de credencialización); el acceso restringido a redes sociales con valor estratégico en el mercado de trabajo; sin contar con aspectos inherentes a nichos segmentados del mercado de trabajo que militan en contra del desarrollo de carreras 
laborales exitosas (alta rotación, bajo premio a la escolaridad, estigmatización, etc.) (Piore, 1975, 1979) $)^{12}$.

Tradicionalmente, en el contexto de la sociedad estadounidense los inmigrantes han mostrado mayor sensibilidad que los nativos a los altibajos económicos, tanto en la fase de declive como de recuperación; en breve, han tendido a comportarse de forma procíclica (Papademetriou y Terrazas, 2010). De acuerdo con datos proporcionados por estos autores, en el lapso comprendido entre el tercer cuatrimestre de 2007 y el tercero de 2009 , el desempleo creció con mayor celeridad en los inmigrantes que en los nativos en al menos doce de diecinueve países europeos, y en Estados Unidos ${ }^{13}$. Solo en dos países se habría expandido a un ritmo inferior en los inmigrantes que en la población nativa (República Checa y Dinamarca) (Papademetriou et al. 2010, p. 41).

Se señala que durante el período recesivo habría tenido lugar una reconfiguración del empleo latino a consecuencia de dos hechos complementarios: la pérdida de peso de la construcción y la industria, y la ganancia en la agricultura y en todas las ramas de los servicios. No obstante, tales sectores - junto al comercio- continuaron alojando el mayor volumen de mano de obra latina en términos relativos (Cachón y AysaLastra, 2015). En sentido general, el peso de la construcción en el empleo inmigrante (masculino) es una constante a nivel internacional. Se estima que 15\% de alrededor de cien millones de migrantes a escala global se aloja en este sector (Martin, 2009, p. 676). En el año 2007, en Estados Unidos había unos tres millones de hispanos trabajando en la construcción (dos tercios de los cuales no habían nacido en dicho país), y prácticamente todos perdieron su empleo (Martin, 2009) ${ }^{14}$. Aunque la contracción de la industria manufacturera fue de menor magnitud que la ocurrida en la construcción, la pérdida de empleos fue de aproximadamente el doble, en virtud del mayor tamaño del sector. Dentro de la manufactura fueron los subsectores de bienes durables los más severamente afectados, en los que la presencia de inmigrantes es proporcionalmente menor (Martin, 2009).

2 Estos aspectos atañen principalmente - aunque no exclusivamente- a los migrantes no calificados, los que constituyen el grueso de la fuerza de trabajo inmigrante latinoamericana.

13 Así habría ocurrido en Austria, Bélgica, Canadá, Estonia, Finlandia, Grecia, Italia, Luxemburgo, Portugal, Suecia y los Estados Unidos (el cálculo incluye a los no ciudadanos). Los autores realizan estas estimaciones con base en un conjunto de fuentes secundarias de los distintos países, y en las estadísticas compiladas por el Eurostat (nota a pie no. 6 de la obra citada).

14 Refiere el autor que luego de que los precios de las casas alcanzaran un récord de 7,7 millones en enero de 2007, se contrajeron a 6,7 en enero de 2009, mientras la tasa de desempleo de los trabajadores en la construcción se disparó de 9\% a 22\% en el mismo lapso (Martin, 2009, p. 677). 
Agricultura y construcción son ámbitos del mercado de trabajo claramente masculinizados, como también algunos subsectores de la manufactura (la producción de automóviles, por ejemplo). El mayúsculo impacto de la crisis en la industria de la construcción y en ciertos sectores productivos de la industria manufacturera ha acaparado la atención de los especialistas. Poco se sabe de lo acontecido con la población femenina inmigrante. De acuerdo con Parella (2015), las mujeres latinoamericanas habrían sido el único grupo que mostró un leve incremento en las tasas de participación económica entre 2007 y 2012, producto sobre todo del desempleo. Al controlar estadísticamente por ciertas características individuales y por factores contextuales (metrópoli de residencia), algunos estudios señalan que en el entorno de la recesión las inmigrantes latinas fueron más afectadas de cara a la probabilidad de encontrar empleo, a diferencia de sus pares masculinos y de las mujeres nacidas en Estados Unidos. El análisis empírico que emprendemos a continuación pretende despejar algunas de estas incógnitas.

\section{Impacto de la crisis en la fuerza de trabajo latinoamericana}

Previo a la descripción de la situación laboral de las inmigrantes latinoamericanas en los años seleccionados (2007, 2009 y 2014), especificamos la fuente de información y la estrategia analítica.

Material y método: fuentes de información y estrategia analítica

La fuente de información en que nos basamos es la Current Population Survey (Encuesta Continua de Población) de Estados Unidos, en su Suplemento Anual Socio Económico de Marzo (ASEC). Dicha encuesta constituye la fuente oficial de estadísticas de empleo y desempleo de Estados Unidos, y es implementada conjuntamente por el Census Burueau y el Burueau of Labor Statistics. Se trata de una encuesta de hogares enfocada a captar el desempleo, las características de la fuerza de trabajo, e información sociodemográfica de toda la población. Se realiza desde hace más de cincuenta años con una periodicidad mensual. Abarca todos los estados y el Distrito de Columbia, y es representativa en los niveles nacional y estatal, como también a escala de subdivisiones geográficas específicas, 
tales como las áreas metropolitanas de Nueva York y Los Ángeles ${ }^{15}$ (Ariza y Caicedo, 2012).

Su principal limitación es el tamaño relativamente reducido de la muestra, lo que impide el análisis a ciertos niveles de desagregación. Como todas las fuentes oficiales de información de Estados Unidos, no indaga sobre el estatus migratorio de los extranjeros, por lo que no es posible identificar a los inmigrantes indocumentados. Con la finalidad de mejorar la confiablidad de las estimaciones referidas a grupos minoritarios, el Sumplemento Social y Económico Anual de Marzo (ASEC), de la Current Population Survey, amplía la muestra original con relación a la que se levanta el resto del año, en particular para los hispanos. Por ello, constituye la fuente de información idónea para estudiar la situación de las inmigrantes latinoamericanas y su desempeño en el mercado de trabajo.

La estrategia que guía el análisis empírico persigue contrastar el desempeño de la fuerza de trabajo femenina en tres momentos clave: el contexto precrisis, el interludio de la recesión y la fase de recuperación macroeconómica (año 2014), con base en el examen de grupos de población analíticamente relevantes: nativas e inmigrantes, y dos subuniversos dentro de la población inmigrante: inmigrantes latinoamericanas e inmigrantes de otra región. Desafortunadamente, por las limitaciones muestrales referidas no es posible desagregar por nacionalidad el grupo de las latinoamericanas, con excepción de algunos colectivos (mexicanos y centroamericanos).

El objetivo del ejercicio empírico es realzar la situación de las latinoamericanas en el conjunto de la inmigración femenina a Estados Unidos y dilucidar si se movieron a corriente (procíclicamente) o contra corriente (contracíclicamente) de la coyuntura económica para acercarnos al rol que jugaron. Aunque el examen se centra en la población femenina trabajadora, se incluye a manera de contexto información sobre los hombres en varios cuadros descriptivos y en el desarrollo de la argumentación, siempre que resulte pertinente. No obstante, al emprender el análisis empírico más preciso el universo se restringe a las mujeres, pues el fuerte impacto de la crisis sobre los hombres obscurecería la comprensión de las particularidades en ellas. Además del análisis bivariado, se exponen con posterioridad los resultados del ajuste de un conjunto de modelos de regresión logística para estimar la probabilidad de caer en el desempleo, de acuerdo con una serie de variables independientes.

15 http://usa.ipums.org/usa/ 


\section{Absorción y expulsión del mercado de trabajo}

Las tasas de participación económica y de desempleo proporcionan una mirada complementaria - aunque insuficiente - de las repercusiones de los ciclos económicos sobre la fuerza de trabajo. Mientras las primeras dan cuenta del porcentaje de personas que forman parte del universo laboral, sea porque andan en busca de trabajo o porque efectivamente lo tienen, la segunda denota la respuesta insuficiente del mercado de trabajo, su incapacidad de absorber a todos los que tratan de incorporarse a él. Si bien desde una mirada de largo aliento son múltiples los factores que inciden en ambos, en una perspectiva de corto plazo como la que nos ocupa, y en aras a la simplificación, ambos indicadores pueden ser vistos como una respuesta de ajuste ante la coyuntura económica: en un caso desde el ángulo de la oferta (participación económica); y en el otro, de la demanda laboral (desempleo). Esto es así porque en los momentos en que se abre paso un período recesivo de envergadura, la elevación de las tasas de desempleo obedece principalmente a la destrucción de puestos de trabajo, antes que al aumento de la oferta laboral o a factores inherentes a la dinámica de los mercados de trabajo ${ }^{16}$. A su vez, las variaciones en la tasa de participación económica pueden denotar, bien el cese de la búsqueda de empleo ante la adversidad del contexto, o la imposibilidad de abandonar el mercado de trabajo porque se carece de soportes institucionales (seguro de desempleo) o sociales (redes), habida cuenta de que en lapsos breves puede darse por sentada la estabilidad en las tasas de crecimiento poblacional.

Una mirada a los niveles de ambos indicadores en el momento precrisis (año 2007, cuadros 1 y 2) muestra tasas de actividad y de desempleo más altas en las mujeres nativas que en las inmigrantes, con diferencias de 4,4 y 0,5 puntos porcentuales, respectivamente, a la vez que una importante heterogeneidad en el universo de las segundas. Destaca el comportamiento laboral inverso entre las latinoamericanas y el resto de las inmigrantes, con tasas de participación superiores en las provenientes de fuera de nuestra región (55,6\% versus 54\%), acompañadas de una menor intensidad del desempleo (3,3 versus 4,5\%). En suma, en el momento precrisis las latinoamericanas mostraban menores niveles de participación económica y de desempleo, tanto respecto de las nativas como de las inmigrantes provenientes de cualquier otra región.

El interludio recesivo alteró este panorama de varias formas. En primer lugar, elevó sustantivamente la tasa de desempleo del conjunto de la fuerza de trabajo femenina, de 4,2 a 7,5, sin apenas modificar su participación económica (de 58,5\% a 58,3\%). Este aspecto

16 Como podría serlo el desempleo friccional. 
corrobora que el ajuste se dio sobre todo del lado de la demanda laboral en la forma de despidos, con la destrucción aproximada de poco más de 1,5 millones de puestos de trabajo ${ }^{17}$. En segundo lugar, la crisis acortó la brecha en los niveles de actividad económica de nativas e inmigrantes (de 4,4 a 3,2 puntos porcentuales), como consecuencia del tránsito de una parte de las nacidas en Estados Unidos hacia la inactividad (decremento de 0,4 en la tasa de actividad), y un leve aumento en los niveles de participación del conjunto de las inmigrantes (aumento de 0,8 en la tasa), debido principalmente al comportamiento de las latinoamericanas. De modo que, la aparente estabilidad de la tasa de actividad femenina en el nivel global esconde comportamientos diferenciales de los grupos de población que la integran (nativas e inmigrantes).

\section{CUADRO 1}

Tasas de actividad económica según condición migratoria, región de origen y sexo. Modelos de regresión logística de la probabilidad de estar desempleada en Estados Unidos, 2007, 2009 y 2014

\begin{tabular}{|c|c|c|c|c|c|c|}
\hline \multirow{2}{*}{$\begin{array}{c}\text { Condición migratoria } \\
\text { y región de origen }\end{array}$} & \multirow{2}{*}{$\begin{array}{c}2007 \\
\text { Hombres }\end{array}$} & \multicolumn{2}{|r|}{2009} & \multicolumn{3}{|c|}{2014} \\
\hline & & Mujeres & Hombres & Mujeres & Hombres & Mujeres \\
\hline Nativos & 69,8 & 59,2 & 68,6 & 58,8 & 65,8 & 57,0 \\
\hline Inmigrantes & 81,2 & 54,8 & 79,5 & 55,6 & 77,7 & 53,9 \\
\hline Latinoamericanos & 84,1 & 54,0 & 82,7 & 55,6 & 81,5 & 54,1 \\
\hline Otra región & 75,4 & 55,6 & 75,6 & 55,5 & 73,6 & 53,8 \\
\hline Total & 71,6 & 58,5 & 70,3 & 58,3 & 67,7 & 56,5 \\
\hline
\end{tabular}

Fuente: elaborado con base en la Current Population Survey (ASEC)

En tercer lugar, si bien la recesión elevó las tasas de desempleo de todas mujeres trabajadoras, invirtió la situación prevaleciente entre nativas e inmigrantes antes de la crisis, pues en marzo de 2009 eran las nacidas fuera de Estados Unidos - y no las nativas - las que ostentaban los mayores niveles de desempleo, con tasas superiores al conjunto de la fuerza de trabajo femenina $(8,6$ versus 7,5). En el subuniverso de las inmigrantes, fueron las latinoamericanas el grupo más severamente afectado, con niveles de desocupación del orden del 11,3\% (cercanos a los de sus pares varones). En contrapartida, las cifras de desocupación de las inmigrantes provenientes de cualquier otra región $(5,9 \%)$ estuvieron bastante por debajo de los valores exhibidos por las nativas $(7,3 \%)$ y el conjunto de la fuerza de trabajo femenina $(7,5 \%)$.

17 Estimaciones propias con base en la CPS (ASEC). 


\section{CUADRO 2}

Tasas de desempleo según condición migratoria, región de origen y sexo.

Estados Unidos, 2007, 2009 y 2014

\begin{tabular}{|c|c|c|c|c|c|c|}
\hline \multirow{2}{*}{$\begin{array}{l}\text { Condición migratoria } \\
\text { y región de origen }\end{array}$} & \multirow{2}{*}{$\frac{2007}{\text { Hombres }}$} & \multicolumn{2}{|r|}{2009} & \multicolumn{3}{|c|}{2014} \\
\hline & & Mujeres & Hombres & Mujeres & Hombres & Mujeres \\
\hline Nativos & 5,4 & 4,3 & 11,1 & 7,3 & 7,7 & 6,4 \\
\hline Inmigrantes & 4,8 & 3,8 & 9,9 & 8,6 & 5,8 & 7,4 \\
\hline Latinoamericanos & 5,4 & 4,5 & 12,2 & 11,3 & 5,7 & 9,2 \\
\hline Otra región & 3,9 & 3,3 & 6,8 & 5,9 & 6,0 & 5,8 \\
\hline Total & 5,2 & 4,2 & 10,9 & 7,5 & 7,3 & 6,5 \\
\hline
\end{tabular}

Fuente: elaborado con base en la Current Population Survey (ASEC)

En 2014, el escenario presenta matices por destacar. Las tasas de desempleo femeninas descienden en apenas un punto porcentual, de 7,5\% a 6,5\% (en contraste con las masculinas que se contraen proporcionalmente más), pero disminuyen más en términos relativos sus niveles de actividad económica (-1,8): de 58,3\% a 56,5\%. Como ha sido señalado por otros autores (Parella, 2015), este aspecto sugiere que el descenso del desempleo observable en 2014 obedeció más al incremento de la inactividad — producto quizás de la ampliación de las trabajadoras desalentadas-, antes que a la creación de empleo. Si esas trabajadoras no hubieran abandonado la fuerza de trabajo, las tasas de desocupación serían superiores ${ }^{18}$. A cinco años (2009-2014) de la fase más aguda de la Gran Recesión, los indicadores de desempleo continuaban muy por encima de los niveles precrisis y las inmigrantes seguían exhibiendo valores superiores a los de las nativas. Resulta llamativa la marcada disparidad interna en el subuniverso de las inmigrantes, con tasas de desempleo del orden del 9,2\%\% en las latinoamericanas, en contraste con las provenientes de cualquier otra región, cuyos niveles de desocupación eran inferiores incluso a los de las trabajadoras nacidas en territorio estadounidense y a la tasa de desempleo del conjunto de la fuerza de trabajo femenina. Estos datos dan cuenta de una mayor sensibilidad (reactividad) de las inmigrantes latinoamericanas a los altibajos del ciclo económico y denotan la acuciante situación en la cual las sumió, vis a vis el resto de las mujeres trabajadoras.

El moderado descenso del desempleo en $2014^{19}$, de la mano de la disminución en la participación económica a pesar de la expansión del producto bruto interno (de

18 Una incógnita por despejar es si uno de los factores detrás del descenso en las tasas de participación económica es la reducción del stock de inmigrantes producto del mayor retorno.

19 Como señalamos, el mes de junio de 2010 marca el inicio del comportamiento ascendente del PIB (diversos autores con base en la NBER). 
2,2\% en 2013), denota el desfase entre la recuperación del crecimiento en términos macroeconómicos y el comportamiento del mercado de trabajo, ya referido. Algunos analistas adjudican tal asincronía a la insuficiente demanda laboral y al clima de profunda desconfianza imperante (Knoop, 2015; Rothstein, 2012). Se trataría de una suerte de letargo de sectores productivos estratégicos a varios años del cataclismo económico. El cuadro 3 recoge el número de semanas continuas de desocupación para ambos sexos y permite ahondar en las secuelas del período recesivo sobre el empleo ${ }^{20}$.

\section{CUADRO 3}

Semanas continuas de desempleo según condición migratoria y región de origen, ambos sexos,

Estados Unidos 2007, 2009, 2014 (porcentajes). Número de semanas continuas de desempleo

\begin{tabular}{lcccc}
\hline 2007 & 30 Menos & De 4 a 26 & 27 y más & Total \\
\hline Nativos & 22,2 & 60,0 & 17,8 & 100,0 \\
Latinoamericanos & 27,4 & 58,4 & 14,2 & 100,0 \\
Otra región & 17,9 & 48,8 & 33,3 & 100,0 \\
\hline Total & 22,5 & 59,2 & 18,3 & 100,0 \\
\hline 2009 & 30 Menos & De 4 a 26 & 27 y más & Total \\
\cline { 2 - 5 } Nativos & 15,2 & 60,4 & 24,4 & 100,0 \\
Latinoamericanos & 16,9 & 64,1 & 19,0 & 100,0 \\
Otra región & 14,1 & 61,8 & 24,1 & 100,0 \\
\hline Total & 15,3 & 60,9 & 23,8 & 100,0 \\
\hline 2014 & 30 Menos & De 4 a 26 & 27 y más & Total \\
Nativos & 14,4 & 51,8 & 33,8 & 100,0 \\
Latinoamericanos & 16,2 & 50,5 & 33,3 & 100,0 \\
Otra región & 17,1 & 47,0 & 35,9 & 100,0 \\
\hline Total & 14,7 & 51,4 & 33,9 & 100,0 \\
\hline
\end{tabular}

Fuente: elaborado con base en la Current Population Survey (ASEC)

Se deduce de dicha información que el ciclo económico iniciado en diciembre de 2007 ha significado un aumento sustancial del desempleo de larga duración, independientemente de la condición migratoria. En 2014, en contraste con 2007, alrededor de una tercera parte de los desocupados pasaba al menos veintisiete semanas consecutivas procurando insertarse en el mercado de trabajo, algo que siete años atrás acontecía a menos de una quinta parte del universo de los desempleados. Es de destacar que como lastre del

20 Por insuficiencia de muestra no es posible desagregar la información por sexo. 
período recesivo, este rasgo afecta por igual a inmigrantes y nativos. En otras palabras, el desempleo se ha hecho más estructural y eso quizás explique parte del descenso observado en las tasas de actividad económica ${ }^{21}$. Por tanto, la disminución del nivel de desocupación ha estado acompañada de su mayor duración, una tendencia que -lejos de ceder - se acentuó en 2014 en relación con la fase recesiva (2009). Volveremos sobre este punto en las reflexiones finales.

\section{El impacto diferencial del ciclo recesivo}

La observación de los cambios en el volumen de población ocupada por rama de actividad proporciona una idea del efecto diferencial de los altibajos económicos sobre los sectores de producción de bienes, y los de distribución y prestación de servicios (comercio y terciario). Como fue referido en la primera parte de este artículo, la alta sensibilidad de los sectores productivos - en particular la construcción y la manufactura- a los vaivenes económicos suscita una rápida respuesta de ajuste, una de cuyas formas más socorrida es la destrucción de empleo. En sentido general, la mayor concentración de la fuerza de trabajo femenina en el sector servicios en virtud de la división sexual del trabajo predominante, le brinda una relativa protección, al menos en los momentos iniciales del período recesivo (Folbre, 2010; Humphries, 1988; Milkman, 1976; Rubery y Rafferty, 2013). Este aspecto resalta la mediación de la segregación por sexo - como eje estructurante del mercado de trabajo- en el posible impacto del ciclo recesivo. Es necesario destacar, no obstante, que la fuerza de trabajo femenina (nativa e inmigrante) no se distribuye de manera homogénea en los distintos subsectores de los servicios o dentro del comercio (minorista y mayorista).

Como figura en el cuadro A.1 del anexo, las inmigrantes están más concentradas en términos relativos en los servicios personales respecto de las nativas, quienes las aventajan en los demás subsectores del terciario. Las latinoamericanas se encuentran proporcionalmente más concentradas en los servicios personales y en la manufactura, si se las compara con las inmigrantes provenientes de cualquier otra región. Estas, a su vez, tienen una presencia relativa bastante mayor en los servicios más calificados, agrupados aquí bajo la categoría "resto de los servicios".

${ }^{21}$ La encuesta en que nos basamos trae como límite superior la categoría de veintisiete semanas y más, por lo que no es posible indagar las diferencias de duración en el desempleo por encima de este umbral. 
El cuadro 4 da cuenta de la variación porcentual entre 2007 y 2014 en el volumen de mujeres ocupadas en un conjunto de ramas de actividad seleccionadas, según subperíodos de análisis. Del mismo pueden colegirse varios aspectos. Entre 2007 y 2009, la mano de obra femenina total ocupada se contrajo $-2,2 \%$, pero esto fue casi con exclusividad resultado de lo ocurrido en el sector manufacturero (en el que incluimos construcción) ${ }^{22}$, pues el comercio apenas se redujo en 2,5\%. Del más de 1,5 millones de mujeres expulsadas, 1,1 eran trabajadoras de dichos sectores. En virtud de las diferencias obvias en el tamaño de estos grupos de población (nativas e inmigrantes), más de 900.000 de los 1,1 millones de empleos destruidos se sustrajeron de las nativas ${ }^{23}$. En contraste con lo acontecido en los sectores de la manufactura/construcción, y el comercio, el sector servicios conservó su presencia relativa, lo que parece dar fe de su comportamiento contra cíclico. En términos comparativos, durante la fase recesiva el universo de la fuerza de trabajo inmigrante (latinoamericana y no latinoamericana) se contrajo proporcionalmente más que la nativa (en al menos 3\%), lo que denota su papel de amortiguación (buffer), de fuerza de trabajo flexible. Entre 2009 y 2014 tuvo lugar una recuperación del volumen de las trabajadoras ocupadas (cuadro 4), con una variación porcentual positiva de 3,1\%. Dicha recuperación obedeció ante todo a la ampliación de la ocupación en el comercio y en el conjunto del terciario, pues la manufactura volvió a contraerse en 2014 respecto de 2009, en cerca de $2 \%(-1,9)$. Es de destacar que la contracción del volumen de mujeres ocupadas en este sector afectó únicamente a las nativas, pues tanto las inmigrantes latinoamericanas como las provenientes de otra región recuperaron parte de su presencia en el sector. Así, en contraste con lo acontecido en la fase aguda de la Gran Recesión, cuando en términos relativos en el conjunto de la fuerza de trabajo ocupada femenina las inmigrantes perdieron más trabajadoras que las nativas, en la fase de recuperación (tomando por esta el año 2014) fueron las inmigrantes (con independencia de la región de origen) las más beneficiadas del proceso de creación de empleos, pues experimentaron incrementos superiores al 10\% en el total de la fuerza de trabajo ocupada respecto de la abrupta caída de 2009 (variación porcentual); las nativas en cambio apenas se mantuvieron $(1,4 \%)$. Es de destacar que fueron los subsectores del comercio (incremento de 5,7\%) y los servicios personales (aumento de 4,3\%) los que lideraron el proceso de reabsorción de fuerza de trabajo ocupada en este subperíodo, seguidos en menor medida del "resto de los servicios" $(2,7 \%)$, ante la atonía mostrada por los sectores de producción de bienes en el período postcrisis, según hemos señalado.

22 Estas ramas han sido agregadas para subsanar los problemas muestrales derivados de la muy baja presencia de las mujeres en la construcción y de que ambos sectores se comportaron procíclicamente.

23 Estimaciones propias con base en la CPS. 


\section{CUADRO 4}

Variación porcentual del volumen de mujeres ocupadas según condición migratoria, región de origen y subperíodo. Ramas de actividad seleccionadas, Estados Unidos

\begin{tabular}{|c|c|c|c|c|}
\hline Subperíodo & & 2007-2009 & & \\
\hline RAMAS & Nativas & Latinoamericanas & Otra región & Total \\
\hline Manufactura y construcción & $-17,9$ & $-18,5$ & $-19,0$ & $-18,0$ \\
\hline Comercio & $-2,6$ & $-5,7$ & 3,7 & $-2,5$ \\
\hline S.Personales & 1,1 & $-3,6$ & 1,0 & 0,5 \\
\hline Resto servicios* & 0,2 & 2,1 & $-1,8$ & 0,1 \\
\hline Total & $-2,0$ & $-3,6$ & $-3,2$ & $-2,2$ \\
\hline Subperíodo & $2009-20$ & & & \\
\hline RAMAS & Nativas & Latinoamericanas & Otra región & Total \\
\hline Manufactura y construcción & $-5,6$ & 14,7 & 13,9 & $-1,9$ \\
\hline Comercio & 5,6 & 5,9 & 6,5 & 5,7 \\
\hline S.Personales & 0,5 & 12,0 & 21,3 & 4,3 \\
\hline Resto servicios* & 1,1 & 12,8 & 15,8 & 2,7 \\
\hline Total & 1,4 & 12,3 & 14,9 & 3,1 \\
\hline Subperíodo & $2007-2$ & & & \\
\hline RAMAS & Nativas & Latinoamericanas & Otra región & Total \\
\hline Manufactura y construcción & $-22,5$ & $-6,5$ & $-7,8$ & $-19,6$ \\
\hline Comercio & 2,8 & $-0,1$ & 10,4 & 3,1 \\
\hline S.Personales & 1,6 & 8,0 & 22,5 & 4,8 \\
\hline Resto servicios* & 1,2 & 15,2 & 13,8 & 2,8 \\
\hline Total & -0 , & 8,3 & 11,2 & 0,8 \\
\hline
\end{tabular}

* Incluye los servicios financieros, los sociales y administración pública

Fuente: Elaborado con base en la Current Population Survey (ASEC)

La franja inferior del cuadro 4 (lapso 2007-2014) permite hacer un balance de los siete años transcurridos desde 2007. Al observar la presencia relativa de las trabajadoras según condición migratoria (total horizontal), queda de manifiesto que el nivel de ocupación global apenas se mantuvo (variación porcentual positiva de $0,8 \%$ ). Fueron las trabajadoras 
inmigrantes las que recuperaron más presencia, pues el volumen de las nativas se encuentra prácticamente en el mismo nivel (aunque algo por debajo, $-0,7 \%$ ), respecto de 2007. Este saldo desfavorable de las nacidas en Estados Unidos es consecuencia del comportamiento del sector manufacturero que - como vimos-continuó contrayéndose en la fase de recuperación (2009-2014). Bien vistas las cosas, en 2014 ninguno de los tres grupos de trabajadoras había recuperado cabalmente la presencia de que gozaba en 2007 en el sector de la manufactura/construcción, aun cuando sin lugar a duda han sido las nativas las más desfavorecidas en términos relativos.

Estos aspectos sugieren la necesidad de indagar a mayor profundidad la configuración (y reconfiguración) del empleo nativo e inmigrante en la manufactura a raíz de la Gran Recesión. En la lógica de los mercados de trabajo segmentados, la mayor rigidez en responder a la dinámica del ciclo económico que muestra el empleo nativo manufacturero puede dar cuenta - por ejemplo- de una restructuración duradera del sector, o expresar simplemente el letargo de los sectores productivos a varios años de iniciado el ciclo de expansión, dado el entorno de incertidumbre. La mayor reactividad del empleo inmigrante, tanto en la fase recesiva como expansiva, reafirma el carácter flexible de esta fuerza de trabajo, y el distinto papel que ambos grupos de trabajadoras (nativas e inmigrantes) desempeñan en la estructura de empleo manufacturero y en el mercado de trabajo en sentido general. Desde el prisma de observación que brinda el intervalo 2007-2014, son los servicios personales el subsector del terciario que más ha ampliado su fuerza de trabajo respecto de 2007. Se trata, no obstante, de un ámbito laboral que aloja a solo 6,8\% de la fuerza de trabajo femenina ocupada (cuadro A.1 del anexo), muy heterogéneo, con ámbitos que se distinguen por la mala calidad de sus condiciones de trabajo ${ }^{24}$, y que probablemente responde más a la oferta que a la demanda laboral.

\section{Determinantes de la probabilidad de ser desempleada}

Describiremos en este apartado los resultados del ajuste de un conjunto de modelos de regresión logística para determinar la probabilidad de caer en el desempleo en cada uno de los tres momentos de observación (2007, 2009 y 2014).

24 Nos referimos a los servicios reproductivos (de cuidado y servicio doméstico). 
La variable dependiente es dicotómica ( $0=$ empleo; $1=$ desempleo $)$. Entre las independientes figuran rasgos sociodemográficos (edad, escolaridad, situación conyugal y raza) ${ }^{25}$ y sociolaborales (grupo de ocupación y rama de actividad). Son ampliamente conocidas la importancia de la edad y la escolaridad en la posibilidad de que las mujeres se inserten en el mercado de trabajo, así como también la sobrerrepresentación de los jóvenes en el universo de los desempleados. Se incluyen el estado civil como un proxy de la situación familiar en virtud de su relevancia en la participación laboral femenina; y la pertenencia racial como variable de control por ser un eje de diferenciación social de suma importancia en la sociedad estadounidense ${ }^{26}$.

En marzo de $2007^{27}$, las cinco variables que más influían en la posibilidad de pasar a engrosar las filas del desempleo eran: pertenecer a los sectores productivos de la construcción/ minería, la agricultura-silvicultura-pesca, y la manufactura ${ }^{28}$, en lugar de formar parte de los servicios más calificados (categoría de contraste); ser de raza negra en vez de blanca; y no estar ni casada ni unida, en oposición a encontrarse dentro de algún tipo de vínculo conyugal. En un orden de magnitud semejante a estas últimas variables - momios de alrededor de 1,6-, figura el hecho de pertenecer al sector comercio. Aun cuando el valor de la razón de probabilidades de formar parte del sector de la construcción es muy alto $(3,3)$, lo que quiere decir que eleva más de tres veces la chance de desempleo en contraste con formar parte del "resto de los servicios" (financieros, sociales y administración pública), la muy baja presencia de las mujeres en dicho sector atenúa el sentido del hallazgo. En el momento precrisis (2007), tanto la agricultura como la manufactura elevan entre $89 \%$ y $76 \%$ la probabilidad de caer en la desocupación respecto de formar parte del subsector de mayor calificación de los servicios (categoría "resto servicios"). Desde nuestro punto de vista, los altos momios (razones de probabilidad) exhibidos por las ramas de la construcción y la agricultura obedecen en parte a su carácter marcadamente estacional.

25 En el cuadro A.2 del anexo figuran las frecuencias relativas de las variables sociodemográficas.

26 Introducir la condición de hispano o no hispano hubiera acarreado problemas de multicolinealidad.

27 Mes en que se recaba la información del Suplemento Social y Económico (ASEC, por sus siglas en inglés), de la CPS.

28 Con la finalidad de facilitar la exposición, en adelante para referirnos a las dos ramas de actividad agrupadas: agricultura, silvicultura y pesca; y construcción y minería, utilizando solo la primera palabra en cada caso. 


\section{CUADRO 5.}

Modelos de regresión logística de la probabilidad de estar desempleada.

Población económicamente activa femenina en Estados Unidos, 2007, 2009 y 2014

\begin{tabular}{|c|c|c|c|}
\hline \multirow{3}{*}{ Variables independientes } & 2007 & 2009 & 2014 \\
\hline & \multicolumn{3}{|c|}{ Razones de probabilidad } \\
\hline & $\operatorname{Exp}(B)$ & $\operatorname{Exp}(B)$ & $\operatorname{Exp}(\mathrm{B})$ \\
\hline \multicolumn{4}{|l|}{$\overline{\text { Edad }}$} \\
\hline 15 a 24 & 1,541 & 1,321 & 1,179 \\
\hline 25 y más* & - & - & - \\
\hline \multicolumn{4}{|l|}{ Escolaridad } \\
\hline Preparatoria o menos & 1,487 & 1,471 & 1,464 \\
\hline Estudios superiores a preparatoria* & - & - & - \\
\hline \multicolumn{4}{|l|}{ Situación conyugal } \\
\hline Casadao unida* & - & - & - \\
\hline No casada o unida & 1,639 & 1,438 & 1,641 \\
\hline \multicolumn{4}{|l|}{ Raza } \\
\hline Blanca* & - & - & - \\
\hline Negra & 1,667 & 1,487 & 1,972 \\
\hline Otra & 1,366 & 1,329 & ** \\
\hline \multicolumn{4}{|l|}{ Condición de migración } \\
\hline No inmigrantes* & - & - & - \\
\hline Inmigrantes latinoamericanas & 0,774 & 1,260 & 1,255 \\
\hline Inmigrantes de otra región & 0,723 & 0,767 & ** \\
\hline \multicolumn{4}{|l|}{ Grupo de ocupación" } \\
\hline No manual* & - & - & - \\
\hline Manual & 1,552 & 1,340 & 1,199 \\
\hline \multicolumn{4}{|l|}{ Rama de actividad económica } \\
\hline Agricultura, silvicultura y pesca & 1,894 & 2,112 & 2,969 \\
\hline Construcción y mineria & 3,395 & 2,469 & 1,794 \\
\hline Manufactura & 1,764 & 2,278 & 1,411 \\
\hline Transporte, comunicaciones, elect y agua & *** & 1,496 & 1,438 \\
\hline Comercio & 1,648 & 1,665 & 1,398 \\
\hline Servicios personales & 1,570 & 1,220 & 1,410 \\
\hline Resto servicios* & - & - & - \\
\hline Constante & 0,014 & 0,032 & 0,027 \\
\hline -2log de la verosimilitud & 15199,450 & 23959,198 & 19786,632 \\
\hline Porcentaje predichos (Overall \%) & 92,9 & 79,1 & 86,6 \\
\hline * Categoria de contraste & $\mathrm{p}<0.05$ & ** No si & nificativa \\
\hline \multicolumn{4}{|c|}{ "No manual: gerentes, profesionales especializados, técnicos y administrativos } \\
\hline Manual: resto de grupos de ocupación & & & \\
\hline
\end{tabular}

Fuente: Elaborado con base en la Current Population Survey (ASEC) 
Ser de raza negra, en lugar de blanca, o estar fuera de un lazo conyugal ("no casada ni unida"), tienen un impacto semejante en la razón de probabilidades (de alrededor de $60 \%$ en relación con la categoría de contraste), lo mismo que pertenecer al sector comercio seguido de los servicios personales. Formar parte de los sectores manuales de la fuerza de trabajo (en lugar de los no manuales), y tener menos de veinticinco años (en oposición a veinticinco y más), elevan poco más de $50 \%$ la chance de integrarse al contingente de desempleadas.

En coherencia con el análisis bivariado, en el momento precrisis (marzo de 2007), ser inmigrante, de Latinoamérica o de cualquier otra región, disminuye entre $28 \%$ y $23 \%$ la probabilidad de caer en el desempleo en contraposición con haber nacido en Estados Unidos; en otras palabras, las nativas eran más proclives a incorporarse al desempleo que las inmigrantes, con independencia de la región de origen de estas (Latinoamérica u otra). Dos años después, en pleno momento recesivo (marzo de 2009), la situación se invierte: ser inmigrante latinoamericana eleva $26 \%$ la probabilidad de caer en el desempleo, en contraste con haber nacido en Estados Unidos. Vale la pena resaltar que en este año se diferencia el comportamiento de las latinoamericanas respecto del "resto de las inmigrantes", pues estas últimas continúan teniendo menor probabilidad de ser desempleadas que las nacidas en la Unión Americana.

En conjunto, los factores sociolaborales muestran bastante fuerza explicativa en 2009, con "momios" superiores a 2 en las ramas de la construcción, la manufactura y la agricultura, seguidas por el sector del comercio (momio de 1,665), lo que quiere decir que formar parte de estos sectores duplica la chance de integrarse a las filas de las desempleadas. En ese año, las variables sociodemográficas que más inciden sobre la razón de probabilidades continúan siendo la raza (raza negra en lugar de blanca), la escolaridad (contar con nivel de preparatoria o menos) y el estado conyugal (estar fuera de algún lazo marital).

Siete años más tarde, en 2014 (y a cuatro años de reiniciado el crecimiento económico), el panorama posee paralelismos y diferencias que destacar. Igual que en los dos años de observación previos, pertenecer a cualquier rama de actividad económica distinta del subsector más calificado de los servicios (categoría de contraste), eleva la probabilidad de desempleo, con diferencias de intensidad pero con una jerarquía semejante. Las variables sociodemográficas de mayor fuerza explicativa son, de nueva cuenta, la pertenencia racial, el estado conyugal y el nivel de escolaridad. Llama la atención que la primera de estas (ser de raza negra en lugar de blanca) casi duplica la chance de caer en el desempleo, dato que realza la fuerte situación de desventaja laboral que aqueja a los afroamericanos en contraste con otros grupos poblacionales, a varios años del interludio recesivo. De 
cara a nuestro interés analítico, el dato más relevante es que en 2014 -al igual que en 2009, pero a diferencia de 2007- las inmigrantes latinoamericanas siguen mostrando una mayor propensión al desempleo que sus homólogas nativas (exponencial de beta de $1,255)$, al tiempo que dejaron de ser estadísticamente significativas las diferencias entre nativas e inmigrantes de otra región respecto de la probabilidad de desempleo.

\section{Consideraciones finales}

El ejercicio empírico llevado a cabo en este trabajo permite vislumbrar algunos de los matices de la compleja relación entre ciclos económicos y fuerza de trabajo femenina, nativa e inmigrante. Aun cuando la Gran Recesión - como otros períodos análogostuvo un impacto mayor en la fuerza de trabajo masculina, no puede soslayarse la severidad con que ellas perdieron puestos de trabajo ni el hecho de que a más de seis años del cataclismo económico no hubieran recuperado las condiciones previas a la crisis. El efecto de la crisis sobre la fuerza de trabajo por sexo está mediado por la segregación sexual del mercado de trabajo: la sobreconcentración de las mujeres en el sector servicios las protegió relativamente en los momentos iniciales de la recesión, pero no las eximió de ser expulsadas con igual vertiginosidad de la manufactura y otros ámbitos productivos. Tampoco de sufrir las consecuencias negativas del desempleo de larga duración como secuela perdurable del intervalo recesivo.

Los distintos segmentos de la fuerza de trabajo femenina considerados mostraron comportamientos disímiles en el contexto de crisis y débil recuperación que le sucedió. En general, las inmigrantes fueron más reactivas a las distintas fases del ciclo, reafirmando su carácter de fuerza de trabajo flexible: resultaron desestimadas en mayor medida en el momento más agudo, pero reabsorbidas también con mayor celeridad en la fase de recuperación (2009-2014); algo que no ocurrió en las nativas, quienes fueron particularmente afectadas por la retracción del empleo manufacturero. Como vimos, en contraste con las inmigrantes, entre 2007 y 2009, una parte de las nacidas en Estados Unidos optó por abandonar el mercado de trabajo (descenso de la tasa de participación económica); alternativa que con seguridad no era igualmente plausible para la población inmigrante, al menos la latinoamericana.

A pesar de la efectiva "reabsorción" o recuperación de una parte del empleo inmigrante entre 2009 y 2014, el ciclo económico iniciado en diciembre de 2007 ha hecho notablemente más crítica la situación de las latinoamericanas, expresada en tasas de 
desempleo elevadas y consistentemente superiores a las de las nativas y las provenientes de cualquier otra región a varios años de concluida la fase recesiva. Es de destacar que esta afectación no hace sino agudizar las peores condiciones laborales que las distinguen en el universo de los inmigrantes (Caicedo, 2010). Como mostró el análisis estadístico multivariado, en contraste con la situación que prevalecía antes de la crisis (marzo 2007), tanto en 2009 como en 2014, las latinoamericanas tenían mayor probabilidad de caer en el desempleo respecto de las nacidas en Estados Unidos. Las inmigrantes de otra región, en cambio, fueron relativamente menos afectadas, con tasas de desocupación inferiores al conjunto de la fuerza de trabajo femenina en los tres momentos de observación.

El moderado descenso de las tasas de desempleo a casi siete años del inicio de la crisis y el carácter más estructural de la desocupación (desempleo de larga duración) traslucen la debilidad del mercado de trabajo en el contexto postcrisis y mueven a la reflexión sobre los límites estructurales que enfrenta la economía capitalista. ¿Por qué las dos recesiones ocurridas en Estados Unidos en el siglo XXI (2001 y 2007) han dado lugar a sendos períodos de recuperación sin creación de empleos (jobless)? Es sabido que las consecuencias del desempleo de larga duración son bastante más devastadoras que las del desempleo cíclico, en virtud de su potencial para socavar los activos de los hogares y desencadenar una espiral de eventos negativos que cancelen las posibilidades de reinserción laboral en el largo plazo. El impacto con que la crisis golpeó a la fuerza de trabajo inmigrante latinoamericana pone en evidencia su mayor vulnerabilidad relativa y realza su carácter flexible en el contexto del mercado de trabajo estadounidense. Resta por conocer cuáles han sido las consecuencias humanas y sociales de la mayor crisis económica de que se tiene noticia desde la Gran Depresión, sobre las trabajadoras latinoamericanas en ese país.

\section{Referencias bibliográficas}

Ariza, M. y Caicedo, M. (2012). Inventario de encuestas e investigaciones cualitativas sobre la migración mexicana a Estados Unidos. México, D.F.: Universidad Nacional Autónoma de México-Instituto de Investigaciones Sociales.

Cachón, L. y Aysa-Lastra, M. (2015). Native and Latino Employment during the Great Recession in the US and Spain. En M. Aysa-Lastra y L. Cachón (Eds.), Immigrant Vulnerability and Resilience: Comparative Perspectives on Latin American Immigrants during the Great Recession (pp. 25-44). New York/London: Springer. 
Caicedo, M. (2010). Migración, trabajo y desigualdad: los inmigrantes latinoamericanos y caribeños en Estados Unidos. México, D.F.: El Colegio de México.

Eichengreen, B. (2015). Hall of Mirrors: The Great Depression, the Great Recession, and the Uses-and Misuses-of History. Berkeley, CA: Oxford University Press.

Fligstein, N. y Goldstein, A. (2011). The Roots of the Great Depression. En D.B. Grusky, B. Western y C. Wimer (Eds.), The Great Recession (pp. 21-56). New York: Russell Sage Foundation.

Folbre, N. (13 de diciembre de 2010). The Declining Demand of Men. The New York Times. Recuperado de https://economix.blogs.nytimes.com/2010/12/13/the-decliningdemand-for-men/?_r=0

Grusky, D.B., Western, B. y Wimer, C. (2011). The Consequences of the Great Recession. En D.B. Grusky, B. Western y C. Wimer (Eds.), The Great Recession (pp. 3-20). New York: Russell Sage Foundation.

Hartmann, H. (2009). Gender Implications of the Financial Crisis in the United States. Washington, DC: Heinrich Böll Foundation North America/Center of Concern/ Institute for Women's Policy Research. Recuperado de https://us.boell.org/sites/ default/files/downloads/Heidi_Hartmann_Paper_Final.pdf

Heath, J. (2012). Lo que indican los indicadores. Cómo utilizar la información estadística para entender la realidad económica de México. México: Instituto Nacional de Estadística y Geografía.

Hout, M. y Cumberworth, E. (2012). The Labor Force and the Great Recession. Stanford, CA: The Russell Sage Foundation/The Stanford Center on Poverty and Inequality.

Hout, M., Levanon, A. y Cumberworth, E. (2011). Job Loss and Unemployment. En D.B. Grusky, B. Western y C. Wimer (Eds.), The Great Recession (pp. 59-81). New York: Russell Sage Foundation.

Humphries, J. (1988). Women's Employment in Restructuring America: The Changing Experience of Women in Three Recessions. En J. Rubery (Ed.), Women and Recession (pp. 20-47). London: Routledge and Kegan Paul Inc. 
Knoop, T.A. (2015). Business Cycle Economics: Understanding Recessions and Depressions from Boom to Bust. Santa Barbara, CA: Praeger.

Levine, Linda. (2009). The Labor Market during the Great Depression and the Current Recession. Congressional Research Service, CRS Report for Congress. Recuperado de https://digital.library.unt.edu/ark:/67531/metadc26169/m1/1/high_res_d/ R40655_2009Jun19.pdf

Martin, P. (2009). Recession and Migration: A New Era for Labor Migration? International Migration Review, 43, 3, 671-691.

Milkman, R. (1976). Women's Work and Economic Crisis: Some Lessons of the Great Depression. Review of Radical Political Economy, 8, 1.

Mitchell, W.C. (1951). What Happens during Business Cycles: A Progress Report. Studies in Business Cycles, 5. New York: National Bureau of Economic Research.

National Bureau of Economic Research. (5 de mayo de 2017). U. S. Business Cycle Expansion and Contraction. Recuperado de http://www.nber.org/cycles/US_Business_Cycle_ Expansions_and_Contractions_20120423.pdf

Papademetriou, D.G., Sumption, M. Terrazas, A., Burkert, C.S., Loyal, S. y Ferrero-Turrión, R. (2010). Migration and Immigrants Two Years after the Financial Collapse: Where Do We Stand? Report for the BBC World Service. Washington, DC: Migration Policy Institute. Recuperado de http://www.migrationpolicy.org/research/migration-andimmigrants-two-years-after-financial-collapse-where-do-we-stand

Papademetriou, D.G. y Terrazas, A. (2010). Immigrant Integration in the U.S. Labor Market: The Recession and its Aftermath. En Bertelsmann Stiftung y Migration Policy Institute (Eds.), Prioritizing Integration: The Transatlantic Council on Migration (pp. 45-60). Bielefeld: Verlag Bertelsmann Stiftung.

Parella, S. (2015). Latin American Women during the Great Recession in the US and Spain. En M. Aysa-Lastra y L. Cachón (Eds.), Immigrant Vulnerability and Resilience: Comparative Perspectives on Latin American Immigrants during the Great Recession (pp. 67-87). New York/London: Springer.

Piore, M.J. (1975). Notes for a Theory of Labor Market Stratification. En R. Edwards, M. Reich y D. Gordon (Eds.), Labor Market Segmentation (pp. 125-150). Lexington, DC: Heath and Company. 
Piore, M.J. (1979). Birds of Passage: Migrant Labor and Industrial Societies. Cambridge: Cambridge University Press.

Rampell, C. (10 de agosto de 2009). The Mancession. New York Times Economic Blog. Recuperado de https://economix.blogs.nytimes.com/2009/08/10/the-mancession/? $\mathrm{r}=0$

Rothstein, J. (2012). The Labor Market four Years into the Crisis: Assessing Structural Explanations. National Bureau of Economic Research Working Paper Series. Universidad de California y NBER. Inédito.

Rubery, J. y Rafferty, A. (2013). Women and Recession Revisited. Work, Employment and Society, 27 (3), 414-432.

Rubery, J. y Tarling, R. (1988). Women's Employment in Declining Britain. En J. Rubery (Ed.), Women and Recession (pp. 100-134). London: Routledge and Kegan Paul.

Rubery, J. (Ed.). (1988). Women and Recession. London: Routledge and Kegan Paul.

Solimano, A. (2015). Elites económicas, crisis y capitalismo del siglo xxi. La alternativa de la democracia económica. México, D.F.: Fondo de Cultura Económica. 


\title{
ANEXO
}

\author{
CUADRO A.1
}

Distribución porcentual de la población ocupada femenina por rama de rama de actividad económica, condición migratoria y región de origen, Estados Unidos, 2007

\begin{tabular}{lcccc}
\hline \multirow{2}{*}{$\begin{array}{l}\text { Rama de } \\
\text { Actividad }\end{array}$} & Condición migratoria y región de origen & & \\
\cline { 2 - 5 } & Nativas & Latinoamericanas & Otra región & Total \\
\hline Agricultura, silvicultura y pesca & 1,4 & 1,4 & 0,8 & 1,3 \\
Manufactura y construcción & 9,0 & 13,7 & 10,9 & 9,5 \\
Transp., comunic. Elect. y agua & 2,7 & 2,7 & 2,7 & 2,7 \\
Comercio & 19,7 & 23,9 & 18,4 & 19,9 \\
Servicios personales & 6,0 & 13,5 & 10,2 & 6,8 \\
Resto de servicios & 61,2 & 44,8 & 57,0 & 59,8 \\
\hline Total & 100,0 & 100,0 & 100,0 & 100,0 \\
\hline
\end{tabular}

Fuente: Elaborado con base en la Current Population Survey (ASEC)

CUADRO A.2

Rasgos sociodemográficos de la población femenina ocupada según condición migratoria y región de origen, Estados Unidos, 2007

\begin{tabular}{lccc}
\hline Variables & Nativas & Latinoamericanas & Otra región \\
Sociodemográficas & & & \\
\hline Edad & 14,7 & 11,5 & 8,4 \\
De 15 a 24 & 55,4 & 66,7 & 62,1 \\
De 25 a 49 & 29,9 & 21,8 & 29,5 \\
\hline 50 y más & 100,0 & 100,0 & 100,0 \\
\hline Total & 35,9 & 65,0 & 29,5 \\
\hline Escolaridad & 64,1 & 35,0 & 70,5 \\
Preparatoria o menos & 100,0 & 100,0 & 100,0 \\
\hline Algún grado superior a Preparatoria & & & \\
\hline Total & 53,5 & 56,7 & 66,9 \\
\hline Estado conyugal & 46,5 & 43,3 & 33,1 \\
\hline Casada o unida & 100,0 & 100,0 & 100,0 \\
\hline No casada o no unida & & & \\
\hline Total & & & \\
\hline
\end{tabular}

Fuente: Elaborado con base en la Current Population Survey (ASEC) 